\title{
Selection of controlled variables: Maximum gain rule and combination of measurements
}

\author{
Eduardo Shigueo Hori and Sigurd Skogestad* \\ Department of Chemical Engineering, \\ Norwegian University of Science and Technology, NTNU, \\ N-7491, Trondheim, Norway
}

November 28, 2007

\begin{abstract}
The appropriate selection of controlled variables is one of the most important tasks in plantwide control. In this paper, we consider the selection of secondary controlled variables for indirect control and the importance of input and output scalings. The objective is to keep the primary variables close to their desired setpoint at steady-state without controlling them directly. We use the maximum scaled gain rule (maximize minimum singular value) and compare it to the exact local method. It is also shown here that the issue of input scaling may be crucial for ill-conditioned plants. Another issue is the selection of optimal combination of variables, where several measurements are combined to reduce the effect of disturbances and implementation error in the primary variables. A two-step methodology to select the combination of variables is evaluated. The methodology is applied to the selection of control structures for a binary distillation column.
\end{abstract}

Keywords: self-optimizing control, distillation columns, plantwide, control system design, disturbance rejection

*To whom correspondence should be addressed. E-mail: skoge@chemeng.ntnu.no. Tel: +47-735-94154. Fax:+47-735-94080 


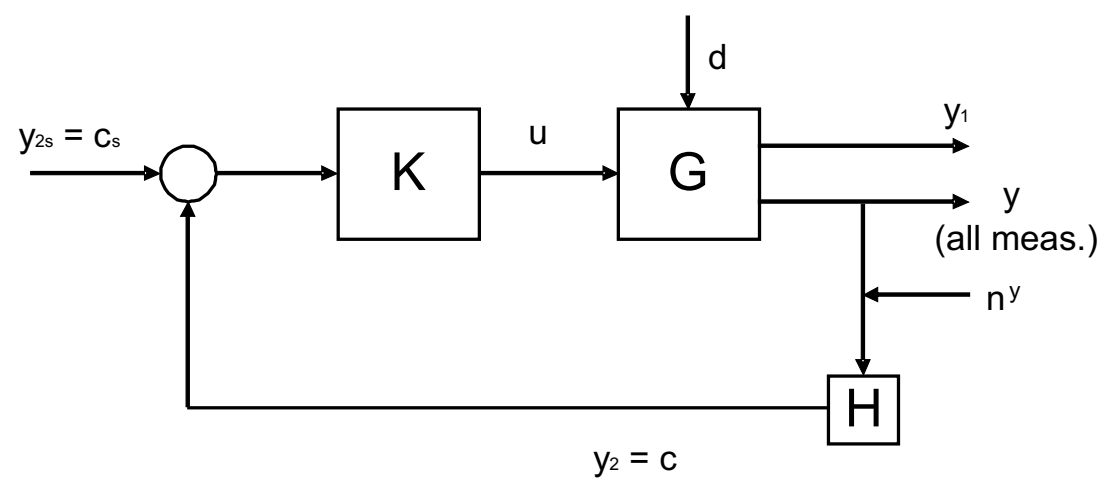

Figure 1: Block diagram of indirect control with selection of measurements.

\section{Introduction}

The selection of controlled variables is one of the most important tasks in control structure design ${ }^{1}$ because this choice can limit the performance of the whole control system. This problem is combinatorial in nature and has been addressed by many authors. ${ }^{2 ; 3 ; 4 ; 5 ; 6}$

For problems where the constraints are active, it is clear that the active constraints should be selected as controlled variables. On the other hand, if the optimum is unconstrained, the choice of controlled variables is much more difficult because there are no limit on the possible variables or combinations.

In this paper, we are concerned with the regulatory control layer, where one issue is to select secondary variables $\left(c=y_{2}\right)$ such that we indirectly achieve good control of the primary variables $\left(y_{1}\right)$ in spite of disturbances (d) and implementation errors $\left(n_{y_{2}}\right)$. Candidate measurements in this paper include temperatures, flows and flow ratios. Figure 1 shows the block diagram representation of indirect control, where matrix $H$ represents the selection/combination of measurements $y$. In the first part of this paper, we consider the common case with control of individual measurements $c$, where $H$ is a selection matrix where each row has one 1 (and the rest 0 's).

The idea of self-optimizing control ${ }^{7}$ was originally formulated by Morari et al., ${ }^{8}$ inspired by the work of Findeisen et al. ${ }^{9}$ It is when keeping the selected variables $c$ constant, indirectly gives acceptable operation or, more precisely, a small loss $L$ in the presence of disturbances and implementation error (without the need of re-optimizing the plant when disturbances occur). Here, the loss $L$ is defined as the difference between the actual value of the cost function obtained with a specific control strategy and the truly optimal value of the cost function, i.e. $L=J-J_{o p t}(d) .{ }^{10}$

For the selection of the controlled variables, $c$, there are some desirable 
properties (requirements) that should be fulfilled: ${ }^{7}$

1. We want a small optimal variation $\Delta c_{\text {opt }}(d)$ to disturbances in the selected variables, i.e. the optimum value of the controlled variables should be insensitive to disturbances.

2. We want variables $c$ with a large sensitivity, ${ }^{3 ; 11}$ or more generally, large gain in terms of the minimum singular value, ${ }^{3} \underline{\sigma}(G)$, from the inputs $u$ to the secondary variables $c$.

3. We want to be able to control the selected controlled variables tightly (small "implementation" error), i.e. they should be easy to measure and control accurately.

Moore $^{3}$ proposed to select controlled variables $c$ using an SVD-analysis of the steady-state gain matrix $G_{\text {all }}$ from the inputs $u$ to all the candidate measurements $y$. After decomposing the gain matrix $G_{\text {all }}=U \Sigma V^{T}$, he proposed to use the orthonormal matrix $U$ (matrix of left singular vectors) to locate the most sensitive measurements (with largest absolute values), which should be used as controlled variables.

These requirements can be conflicting, as there is a trade-off between high gain and small implementation error, Halvorsen et al. ${ }^{10}$ derived rigorously the closely related method of selecting controlled variables that maximize the minimum singular value, $\underline{\sigma}\left(G^{\prime}\right)$, of the appropriately scaled gain matrix from inputs $u$ to the selected outputs $c$. The "maximum gain rule" ${ }^{10}$ is: Select controlled variables $c$ such that we maximize the minimum singular value of the scaled gain matrix $G^{\prime}, \underline{\sigma}\left(G^{\prime}\right)$, where

$$
G^{\prime}=S_{1} G S_{2}
$$

Here, $G$ is the steady-state gain matrix from $u$ (manipulated variables) to $c$ (controlled variables). $S_{1}$ and $S_{2}$ are the output and input scalings, respectively.

The output scalings $S_{1}$ are obviously important as the objective is to select the outputs (controlled variables), and the output scalings indirectly include the control objective through the optimal variation, see Eq. (8). However, the input scaling has been simplified by assuming that $S_{2}$ is a diagonal or unitary matrix. ${ }^{10}$ This assumption may seem to be of minor importance because the inputs are anyway given. The main goal of this paper is to reexamine this assumption.

To do this, we compare the maximum gain rule with the exact local method $^{10}$ on a binary distillation column, where $u=\left[\begin{array}{ll}L & V\end{array}\right], y_{1}=\left[\begin{array}{ll}x_{\text {top }}^{H} & x_{\text {btm }}^{L}\end{array}\right]$ and $c=H y$ is a combination of temperatures and/or flows. The distillation 
column was selected because it is "ill-conditioned" with a large variation in gain depending on the input direction, and the scaling $S_{2}$ is expected to be more critical in such cases. The objective function $J$ to be minimized is the relative steady-state deviation from the desired setpoint,

$$
J=\Delta X^{2}=\left(\frac{x_{t o p}^{H}-x_{t o p, s}^{H}}{x_{t o p, s}^{H}}\right)^{2}+\left(\frac{x_{b t m}^{L}-x_{b t m, s}^{L}}{x_{b t m, s}^{L}}\right)^{2}
$$

where $x_{t o p}^{H}$ is the composition of the heavy key-component $(\mathrm{H})$ in the top of the column and $x_{b t m}^{L}$ is the composition of the light key-component (L) in the bottom.

The second part of this paper is related to selecting the optimal combination of variables that minimizes the effects of disturbances and implementation error. ${ }^{6 ; 12 ; 13}$ Here, we use the two-step procedure of Alstad and Skogestad. ${ }^{13}$ In the first step, we apply the Maximum Gain Rule to select the best set of measurements to be used for indirect control. In the second step, we obtain the matrix $H$ in order to reduce the effect of disturbances and implementation error. Hori et al. ${ }^{12}$ presented a simple way to evaluate matrix $H$ in order to achieve perfect indirect control (zero disturbance loss). The same result was obtained by Alstad and Skogestad ${ }^{13}$ using the null space method. The problem with these methods is that they do not include the implementation error. Alstad et al. ${ }^{14}$ derived an analytical solution for the exact local method to find the optimal combination of measurements $H$ for the case with combined disturbances and measurement errors, and all these methods are compared on the distillation case study.

\section{Maximum gain rule}

In the Maximum Gain Rule, the objective is to select controlled variables to maximize the minimum singular value of $G^{\prime}=S_{1} G S_{2}$. The minimum singular value has the monotonic property, which means that we can use a branch and bound algorithm to search for the configuration with largest minimum singular value, thus avoiding the evaluation of all possible configurations. ${ }^{15}$

To evaluate the maximum gain rule, and in particular the effect of the scaling $S_{2}$, we define the loss $L$ as the difference between the actual value of the cost function $J(u, d)$, obtained with a specific control strategy, e.g. with the controlled variable $c$ is constant, and the truly optimal value of the cost function $J_{\text {opt }}(d)$, that is,

$$
L(u, d)=J(u, d)-J_{\mathrm{opt}}(d)
$$


In our case, with indirect control, $J_{\text {opt }}(d)=0$, see Eq. (2).

The second-order expansion of the loss function is ${ }^{10}$

$$
L(u, d)=\frac{1}{2}\left(c-c_{\text {opt }}(d)\right)^{T} G^{-T} J_{u u} G^{-1}\left(c-c_{\text {opt }}(d)\right)
$$

where $J_{u u}$ is the matrix of second derivatives of $J$ (Hessian matrix) and $G$ is the gain matrix from inputs $u$ to $c$.

Defining $z=J_{u u}^{-1 / 2} G^{-1}\left(c-c_{\text {opt }}(d)\right)$, Eq. (4) becomes $^{10}$

$$
L(u, d)=\frac{1}{2}\|z\|_{2}^{2}
$$

Introducing $e_{c}=c-c_{o p t}(d)$ and assuming that each controlled variable $c_{i}$ is scaled such that the sum of its optimal range $\left(c_{\text {opt }}\right)$ and its implementation error $\left(n^{y}\right)$ is unity, i.e., for combined errors the 2-norm is less or equal to 1 , Halvorsen et al. ${ }^{10}$ showed that the worst-case loss is

$$
L_{\max }=\max _{\left\|e_{c}^{\prime}\right\|_{2} \leq 1} \frac{1}{2}\|z\|_{2}^{2}=\frac{1}{2\left(\underline{\sigma}\left(S_{1} G J_{u u}^{-1 / 2}\right)\right)^{2}}
$$

The (exact) maximum gain rule is then to maximize $\underline{\sigma}\left(S_{1} G S_{2}\right)$, where $S_{2}=J_{u u}^{-1 / 2}$.

\subsection{Maximum gain rule: Output scaling $\left(S_{1}\right)$}

An important part of the maximum gain rule is to scale the output variables appropriately, i.e., such that $\left\|e_{c}^{\prime}\right\|_{2} \leq 1$ holds. The outputs are scaled with respect to their "span", which is the sum of

1. optimal variation $\left(c_{o p t}\right)$ due to disturbances $d$

2. effect of implementation error $\left(n^{y}\right)$

and we have

$$
S_{1}=\operatorname{diag}\left\{1 / \operatorname{span}\left(c_{i}\right)\right\}
$$

where

$$
\operatorname{span}\left(c_{i}\right)=\mid \text { opt. var. }|+| \text { implem. error }\left.|=| c_{\text {opt }}\right|_{i}+\left|n^{y}\right|_{i}
$$

The optimal variation may be obtained as follows. Write the linear steadystate model as:

$$
\begin{gathered}
y_{1}=G_{1} u+G_{d 1} d \\
c=G u+G_{d} d
\end{gathered}
$$


where $y_{1}$ are the primary variables, $c$ are the measurements (candidate controlled variables), $u$ are manipulated variables and $d$ are disturbances. In the presence of disturbances $(d)$, perfect control of the primary variables $\left(y_{1}=0\right)$ is obtained with

$$
u_{\mathrm{opt}}=-G_{1}^{-1} G_{d 1} d
$$

The resulting optimal variation of the measurements $(c)$ is

$$
c_{\mathrm{opt}}=\left(-G G_{1}^{-1} G_{d 1}+G_{d}\right) d
$$

Remark: We here use perfect control $\left(y_{1}=0\right)$ as the reference for computing the optimal variation. This is recommended even for cases where some manipulated variables are kept constant (e.g. reflux $L$ is constant) such that $y_{1}=0$ is not possible, because perfect control of $y_{1}$ is in any case the objective.

\subsection{Maximum gain rule: Input scaling $\left(S_{2}\right)$}

From Eq. (6), the best (correct) input "scaling" for the maximum gain rule is to select $S_{2}=J_{u u}^{-1 / 2}$, where $J_{u u}$ is the Hessian matrix of the cost function $J$ (matrix of second derivatives of $J$ with respect to $u$ ). The term "scaling" is a bit misleading because $J_{u u}^{-1 / 2}$ is generally not a diagonal matrix. In many cases, ${ }^{10}$ it is assumed that $S_{2}=J_{u u}^{-1 / 2}$ is unitary, which is referred to as simplified scaling.

\subsection{1 $S_{2}=J_{u u}^{-1 / 2}$ (correct)}

From Eq. (6), the correct maximum gain rule states that, in order to minimize the loss $L$, we need to find the set of controlled variables $c$ that maximize $\underline{\sigma}\left(S_{1} G J_{u u}^{-1 / 2}\right)$.

\subsubsection{Simplified scaling (assuming $J_{u u}$ unitary)}

For the minimum singular value, the property $\underline{\sigma}(A B) \geq \underline{\sigma}(A) \cdot \underline{\sigma}(B)$ holds for any non-singular matrices $A$ and $B$ of any dimension. ${ }^{16}$ So, from Eq. (6), we have that $\underline{\sigma}\left(G^{\prime}\right)=\underline{\sigma}\left(S_{1} G J_{u u}^{-1 / 2}\right) \geq \underline{\sigma}\left(J_{u u}^{-1 / 2}\right) \underline{\sigma}\left(S_{1} G\right)$. As $\underline{\sigma}\left(J_{u u}^{-1 / 2}\right)=$ $1 / \bar{\sigma}\left(J_{u u}\right)^{1 / 2}$, we derive from Eq. (6) an upper bound for the maximum loss

$$
L_{\max }=\max _{\left\|e_{c}^{\prime}\right\|_{2} \leq 1} L \leq \frac{\bar{\sigma}\left(J_{u u}\right)}{2\left(\underline{\sigma}\left(S_{1} G\right)\right)^{2}}
$$

To analyze the tightness of this bound, we derive a lower bound from the inequality ${ }^{16} \bar{\sigma}(A B) \geq \underline{\sigma}(A) \cdot \bar{\sigma}(B)$. From Eq. (6), we then have $\bar{\sigma}\left(S_{1} G J_{u u}^{-1 / 2}\right) \geq$ 
$\underline{\sigma}\left(J_{u u}^{-1 / 2}\right) \bar{\sigma}\left(S_{1} G\right)$, and using $\bar{\sigma}\left(S_{1} G\right)=1 / \underline{\sigma}\left(S_{1} G\right)$, we then find

$$
\frac{\underline{\sigma}\left(J_{u u}\right)}{2\left(\underline{\sigma}\left(S_{1} G\right)\right)^{2}} \leq L_{\max } \leq \frac{\bar{\sigma}\left(J_{u u}\right)}{2\left(\underline{\sigma}\left(S_{1} G\right)\right)^{2}}
$$

That is, for systems that can be scaled such that $J_{u u}$ has a small condition number $\left(\gamma\left(J_{u u}\right)=\bar{\sigma}\left(J_{u u}\right) / \underline{\sigma}\left(J_{u u}\right)\right)$, the lower and upper bounds become close to each other and the simplified minimum singular method is guaranteed to apply. In Eq. (13), we have equality when we assume that $J_{u u}$ is a unitary matrix (simplified scaling) because then $\bar{\sigma}\left(J_{u u}\right) / \underline{\sigma}\left(J_{u u}\right)=1$.

\section{Exact local method}

To evaluate the maximum gain rules presented in the last section, we calculate the maximum loss for each set of controlled variables using the exact local method. ${ }^{10}$ This method utilizes a Taylor series expansion of the loss function, and the exact value of the worst-case local loss becomes

$$
L_{\max }=\max _{\left\|e_{c}^{\prime}\right\|_{2} \leq 1} L=\frac{\left(\bar{\sigma}\left(\left[\begin{array}{ll}
M_{d} & M_{n^{y}}
\end{array}\right]\right)\right)^{2}}{2}
$$

where

$$
\begin{gathered}
M_{d}=J_{u u}^{1 / 2}\left(J_{u u}^{-1} J_{u d}-G^{-1} G_{d}\right) W_{d} \\
M_{n^{y}}=J_{u u}^{1 / 2} G^{-1} W_{n}
\end{gathered}
$$

$M_{d}$ represents the loss in the primary variables caused by disturbances and $M_{n^{y}}$ represents the loss caused by implementation error.

The magnitude of the disturbances and implementation error enter into the diagonal matrices $W_{d}$ and $W_{n}$, respectively. The steady-state gains $G$ and $G_{d}$ and the second order derivatives $J_{u u}$ and $J_{u d}$ may be obtained numerically by applying small perturbations in the inputs $u$.

In our case, we are interested in indirect control of the top and bottom compositions (see cost function for the distillation column in Eq. (2)), and $J_{u u}$ and $J_{u d}$ can be obtained analytically.

More generally, consider a quadratic cost function

$$
J=y_{1}^{T} Q y_{1}+u^{T} R u
$$

where $Q$ and $R$ are weighting matrices (both are symmetric positive-definite). From Eq. (9), we then have

$$
J_{u u}=2\left(G_{1}^{T} Q G_{1}+R\right)
$$




$$
J_{u d}=2 G_{1}^{T} Q G_{d 1}
$$

In our case, as the cost function is defined by Eq. (2), the weighting matrices are $Q=\operatorname{diag}\left(\left[1 /\left(x_{t o p, s}^{H}\right)^{2} \quad 1 /\left(x_{b t m, s}^{L}\right)^{2}\right]\right)$ and $R=0$.

Compared to the exact method, the branch and bound algorithm for minimizing $\underline{\sigma}\left(G^{\prime}\right)$ usually requires the evaluation of fewer combinations, and each evaluation is also less time consuming.

\section{Case study: Distillation column}

The variable selection methods (maximum gain rule and exact local method) are applied to a binary distillation column. The chemical components are assumed "ideal" and denoted L (light) and H (heavy). It is assumed that the relative volatility is constant $\left(\alpha_{\mathrm{LH}}=1.5\right)$. The main disturbances are the feed flow rate $(F)$, feed enthalpy $\left(q_{F}\right)$, and feed composition $\left(z_{F}\right)$. The example is "column A" 17 with a feed of $50 \%$ mol of light component. The objective of the column is to keep $1 \%$ of heavy component in the top (and $99 \%$ lights) and $1 \%$ of light component in the bottom. The column has 41 stages (including the reboiler and the total condenser) and these stages are numbered from bottom to top (see Figure 2).

A conventional distillation column with a given feed and pressure controlled using cooling has 4 degrees of freedom left: reflux flow rate $(L)$, vapor boilup $(V)$, distillate flow rate $(D)$, and bottoms flow rate $(B)$, i.e., $u_{0}=\left[\begin{array}{llll}L & V & D & B\end{array}\right]^{T}$. We need to control two liquid levels to stabilize the column. This consumes two degrees of freedom because levels do not have steady-state effect and we are left with two steady-state degrees of free$\mathrm{dom}^{18}$ for composition control, which are here selected as $u=\left[\begin{array}{ll}L & V\end{array}\right]$. Note that the steady-state gain matrix $G_{1}$ from $u=\left[\begin{array}{ll}L & V\end{array}\right]$ to $y_{1}$ is generally ill-conditioned. In our case, the gain matrix is $G_{1}=\left[\begin{array}{ll}1.085 & -1.098 \\ 0.875 & -0.862\end{array}\right]$, so

$$
J_{u u}^{-1 / 2}=\left[\begin{array}{ll}
0.263 & 0.259 \\
0.259 & 0.262
\end{array}\right]
$$

The condition number $\gamma=\bar{\sigma} / \underline{\sigma}$ is the same for both $G_{1}$ and $J_{u u}^{-1 / 2}$ in this case, $\gamma\left(G_{1}\right)=\gamma\left(J_{u u}^{-1 / 2}\right)=145.6$ (they are the same because $R=0$ and $Q=\beta I$, where $\beta$ is any constant different from zero). Note that an unitary matrix has a condition number of 1 , so $J_{u u}$ is far from unitary. The minimized condition number with respect to diagonal scaling is also large, $\gamma^{*}\left(G_{1}\right)=$ $\min _{D_{1}, D_{2}} \gamma\left(D_{1} G_{1} D_{2}\right)=141.9$. 


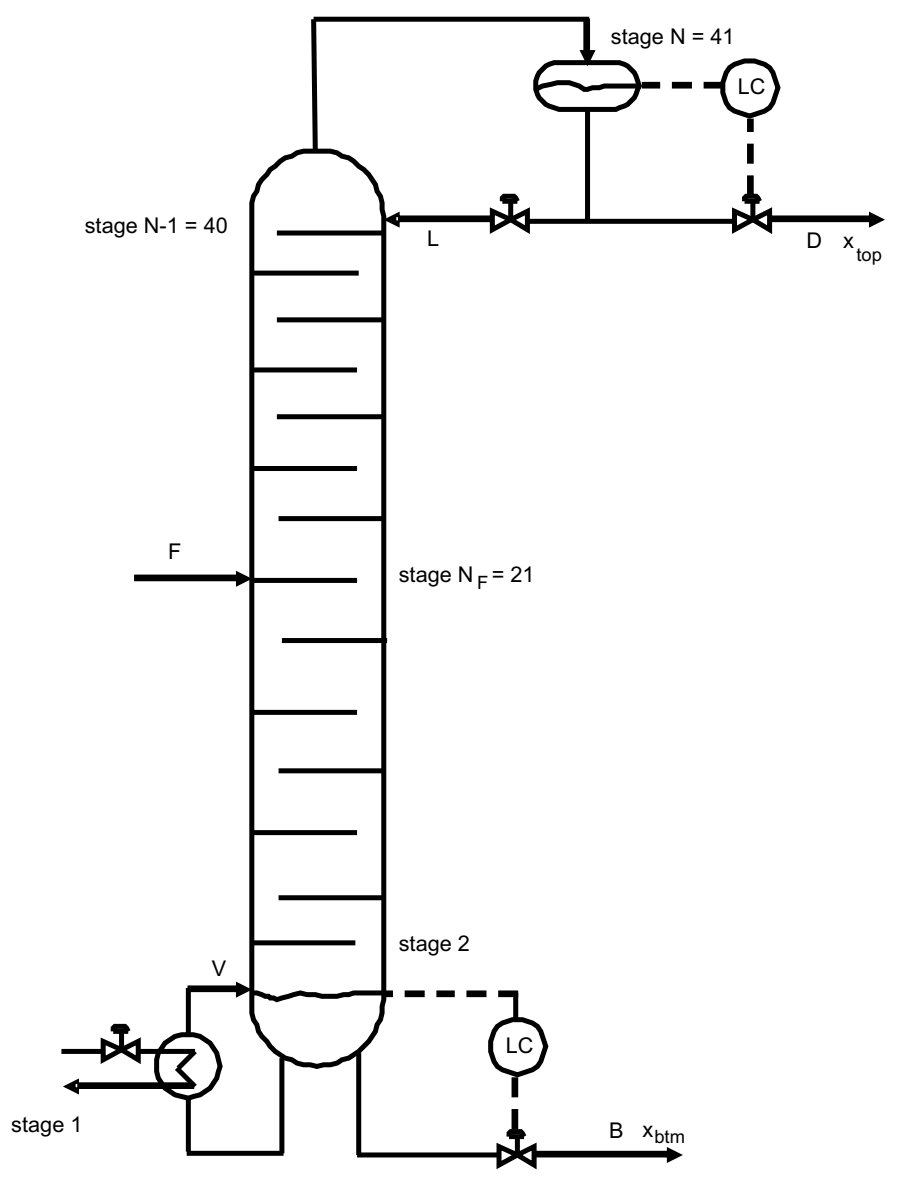

Figure 2: Distillation column. 
Our objective is to use the two available degrees of freedom to keep the top and bottom compositions (primary variables $y_{1}$ ) close to their optimal values, see Eq. (2). As compositions are difficult to measure (due to long time delays, high cost, etc.), we want to use indirect control where temperatures and/or flows (including flow ratios $L / D, L / F, V / B, V / F$ ) are used as secondary controlled variables $(c)$. For simplicity, we assume that the temperature $T_{i}\left({ }^{\circ} \mathrm{C}\right)$ on each stage $i$ can be calculated as a linear function of the liquid composition in each stage ${ }^{19}$

$$
T_{i}=0 x_{A, i}+10 x_{B, i}
$$

This may seem unrealistic, but results using detailed models show that this is actually of minor importance. ${ }^{20}$ To compare the maximum gain rule with the exact method, we use the maximum composition deviation: ${ }^{17}$

$$
\Delta X_{\max }=\sqrt{J_{\max }}=\sqrt{L_{\max }}
$$

where $L_{\max }$ is calculated from Eq. (15) (exact method) or estimated from Eqs (6) (maximum gain rule) and (13) (simplified maximum gain rule).

\subsection{Output scaling $\left(S_{1}\right)$}

The output scaling is $S_{1}=1 / \Delta c_{o p t}$ where

$$
\Delta c_{o p t}=\left|\frac{d c_{o p t}}{d z_{F}}\right| \Delta z_{F}^{E}+\left|\frac{d c_{o p t}}{d F}\right| \Delta F^{E}+\left|\frac{d c_{o p t}}{d q_{F}}\right| \Delta q_{F}^{E}+\left|\Delta c^{n}\right|
$$

Here, $\Delta z_{F}^{E}, \Delta F^{E}$, and $\Delta q_{F}^{E}$ are the expected (typical) disturbances and $\Delta c^{n}$ is the expected implementation/measurement error for controlling the measurements. For temperatures, the implementation error $\left(\Delta c^{n}=\Delta T^{n}\right)$ is assumed to be the same for all stages $\left(\Delta T^{n}= \pm 0.5^{\circ} \mathrm{C}\right)$, while the implementation error is $\pm 10 \%$ for flow rates and $\pm 15 \%$ for flow ratios. The expected magnitude of the disturbances are $20 \%$ for feed rate $F, 10 \%$ for feed composition $z_{F}$ and $10 \%$ for feed enthalpy $q_{F}$.

The resulting optimal variations for temperatures along the column obtained using Eq. (12) are shown in Figure 3. Temperatures on stages close to the feed stage are more sensitive to disturbances, while the stages close to the ends are affected more by the implementation error (the optimal variation is zero at the ends). Also, the figure shows that the main disturbance is in the feed composition. The optimal variation in temperature with feed flowrate is zero, because we have assumed constant efficiency for the column. This result confirms what is found in the literature. ${ }^{21}$ 


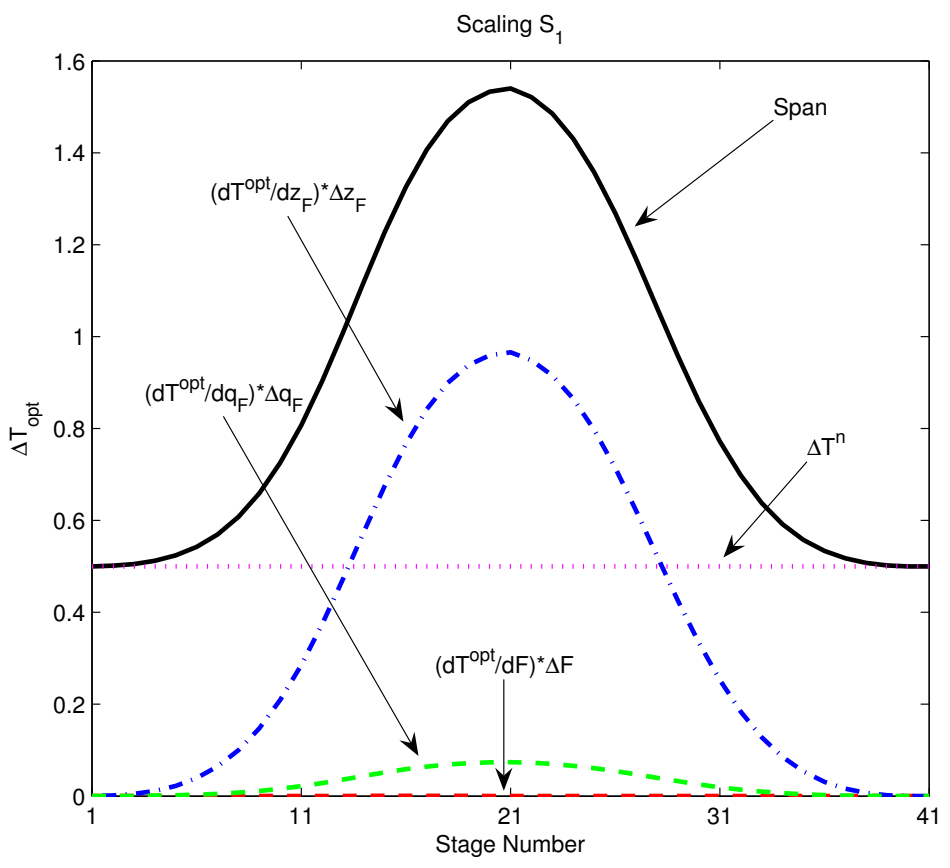

Figure 3: Optimal variations and total span (output scaling $S_{1}$ ).

\subsection{Input scaling $\left(S_{2}\right)$}

In this section, we want to compare the maximum gain rule (section 2.2.1) and the simplified maximum gain rule (section 2.2.2) with the exact local method (section 3). The three methods were compared using the composition deviations estimated by Eqs (6) (maximum gain rule, $S_{1} G J_{u u}^{-1 / 2}$ ), (13) (simplified maximum gain rule, $S_{1} G$ ), and (15) (exact local method). Figure 4 shows the results with two symmetrically located temperatures as controlled variables. A "good" location should have a composition deviation $\Delta X$ of about 1 or less. This figure shows that the three methods give almost the same best temperatures, but note that the estimated $\Delta X$ is a factor 100 times higher when we use $\underline{\sigma}\left(S_{1} G\right)$. This happens because, as shown earlier, the condition number for this process is very large (145.6).

In Table 1, we consider the more general case where the candidates two controlled variables are any combination of temperatures and flows (including flow ratios $L / D, L / F$, etc). Table 1 shows that the simplified maximum gain rule using $\underline{\sigma}\left(S_{1} G\right)$ gives again completely wrong (too high) estimates for $\Delta X$ in most cases. The simplified method gives that the best control configuration with the largest value of $\underline{\sigma}\left(S_{1} G\right)$ is to keep $L / F$ and $V / B$ constant. However, this is actually a poor choice with an exact loss of 18.60 . 
Table 1: Steady-state composition deviation $\Delta X$ for distillation column for various configurations.

\begin{tabular}{|c|c|c|c|c|c|}
\hline \multirow{3}{*}{$\begin{array}{l}\text { Configuration } \\
\text { Fixed variables }(c)\end{array}$} & \multirow{3}{*}{$\begin{array}{l}\text { Exact method } \\
\Delta X(E q .15)\end{array}$} & \multicolumn{4}{|c|}{ Maximum gain rule } \\
\hline & & \multicolumn{2}{|c|}{$\begin{array}{c}\text { Assume } J_{u u} \text { unitary } \\
\text { (simplified) }\end{array}$} & \multicolumn{2}{|c|}{$\begin{array}{c}S_{2}=J_{u u}^{-1 / 2} \\
(\text { correct })\end{array}$} \\
\hline & & $\underline{\underline{\sigma}}\left(S_{1} G\right)$ & $\begin{array}{l}\text { Est. } \Delta X \\
\text { (Eq. } 13) \\
\end{array}$ & $\underline{\underline{\sigma}}\left(S_{1} G J_{u u}^{-1 / 2}\right)$ & $\begin{array}{l}\text { Est. } \Delta X \\
\text { (Eq. } 6)\end{array}$ \\
\hline$T_{12}-T_{30}$ & 0.530 & 1.508 & 131 & 0.783 & 0.903 \\
\hline$T_{12}-T_{29}$ & 0.541 & 1.442 & 137 & 0.752 & 0.941 \\
\hline$T_{14}-T_{28}$ & 0.595 & 1.241 & 159 & 0.645 & 1.100 \\
\hline$T_{9}-T_{32}$ & 0.675 & 1.548 & 127 & 0.792 & 0.893 \\
\hline$T_{15}-T_{26}$ & 0.706 & 0.956 & 206 & 0.499 & 1.417 \\
\hline$T_{15}-L / F$ & 0.916 & 1.531 & 129 & 0.607 & 1.164 \\
\hline$T_{16^{-}} V / F$ & 1.148 & 1.125 & 175 & 0.498 & 1.419 \\
\hline$T_{19}-L$ & 1.223 & 0.815 & 242 & 0.400 & 1.767 \\
\hline$T_{15}-L / D$ & 1.321 & 0.727 & 272 & 0.342 & 2.067 \\
\hline$T_{22}-V$ & 1.470 & 0.639 & 309 & 0.305 & 2.320 \\
\hline$T_{24^{-}}-V / B$ & 1.711 & 0.571 & 345 & 0.261 & 2.712 \\
\hline$T_{1}-T_{41}$ & 5.000 & 0.271 & 728 & 0.141 & 5.000 \\
\hline$L / D-V / B$ & 15.80 & 0.878 & 225 & 0.040 & 17.80 \\
\hline$L / F-V / B$ & 18.60 & 1.603 & 123 & 0.028 & 25.60 \\
\hline$L-B$ & 21.10 & 0.805 & 245 & 0.020 & 35.20 \\
\hline$D-V$ & 21.20 & 0.634 & 311 & 0.020 & 35.20 \\
\hline$L / F-V / F$ & 90.00 & 1.600 & 124 & 0.007 & 109.0 \\
\hline
\end{tabular}




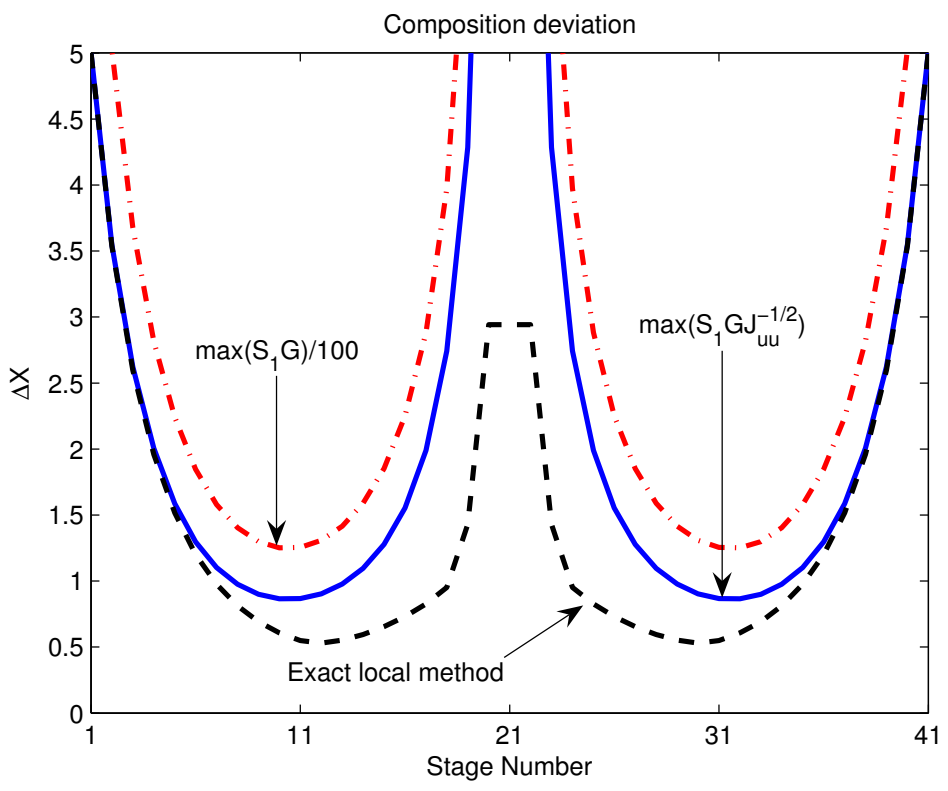

Figure 4: Comparison of composition deviation estimates $\left(S_{1} G J_{u u}^{-1 / 2}, S_{1} G\right.$, and exact local method).

On the other hand, with the correctly scaled gain matrix $G^{\prime}=S_{1} G J_{u u}^{-1 / 2}$, the results with the maximum gain rule are very close to the exact method, giving $T_{9}-T_{32}$ as the proposed best set of controlled variables (exact loss of 0.675). This is close to the minimum steady-state composition deviation $(\Delta X)$ of 0.530 , which is obtained when we control temperatures on stages 12 and 30, that is, with the temperatures symmetrically located on each side of the feed stage. Thus, although the maximum gain rule using $G^{\prime}=S_{1} G J_{u u}^{-1 / 2}$ is not exact, it gives results in terms of temperature selection that are very similar to the exact method. It is thus suitable for screening of candidate controlled variables.

\section{Linear combination of measurements}

So far we have considered the selection of single measurements. Another option is to use combinations of measurements $c=H y$, where $H$ is a combination rather than a selection matrix. The goal of using several measurements is to further reduce the effect of disturbances and implementation errors. As the number of possible measurements is usually very large, it is necessary to select the best set of measurements that minimize the loss. The issue of using multiple temperatures to control distillation columns has been described in 
other papers. ${ }^{22 ; 23}$

\section{$5.1 \quad$ Two-step procedure}

To avoid the evaluation of the exact loss (Eq. 15) for all possible combinations of measurements, Alstad et al. ${ }^{14}$ suggest a two-step approach to obtain the combination of variables.

1. Choose the set of measurements applying the "Maximum Gain Rule" presented by Alstad et al., ${ }^{14}$ i.e., maximize the minimum singular value of the scaled matrix $\tilde{G}^{\prime}=S_{1} \tilde{G}=\left[\begin{array}{ll}S_{1} G & S_{1} G_{d}\end{array}\right]$.

2. The combination matrix $H$ can be evaluated in two different ways:

- Perfect disturbance rejection ${ }^{12}$

$$
H=\tilde{G}_{1} \tilde{G}^{\prime-1}
$$

where $\tilde{G}_{1}=\left[\begin{array}{ll}G_{1} & G_{d 1}\end{array}\right]$.

- Exact local method: Minimize the combined effect of disturbances and implementation error by finding the matrix $H$ that minimize the 2-norm of $M=\left[\begin{array}{ll}M_{d} & M_{n}\end{array}\right]$ in Eq. (15). It can be shown that this is equivalent to minimizing $\|H \tilde{F}\|_{2}^{2}$ subject to $H G^{y}=J_{u u}^{1 / 2}$, where $\tilde{F}=\left[\begin{array}{ll}F W_{d} & W_{n^{y}}\end{array}\right]$ and $F=-\left(G^{y} J_{u u}^{-1} J_{u d}-G_{d}^{y}\right)$. One finds ${ }^{14}$

$$
H^{T}=\left(\tilde{F} \tilde{F}^{T}\right)^{-1} G^{y}\left(G^{y T}\left(\tilde{F} \tilde{F}^{T}\right)^{-1} G^{y}\right)^{-1} J_{u u}^{1 / 2}
$$

Note that the solution in Eq. (26) is not unique, so if $H$ is an optimal solution, another optimal solution is $H_{1}=D H$, where $D$ is a non-singular matrix of dimension $n_{u} \times n_{u}$.

For the LV-distillation column case study, we consider only temperatures as possible measurements and we do not consider the feed flowrate as a disturbance because its optimal variation in temperature is zero (see Section 4.1). Then, we have 2 inputs $\left(n_{u}=2\right), 2$ disturbances $\left(z_{F}, q_{F}\right.$, i.e. $\left.n_{d}=2\right)$, and we need to select 4 temperatures $\left(n_{y}=4\right)$ to obtain perfect disturbance rejection.

\subsubsection{Step 1: selection of measurements}

The heuristic of maximizing $\bar{\sigma}\left(\tilde{G}^{\prime}\right)$, where $\tilde{G}^{\prime}=S_{1} \tilde{G}=\left[\begin{array}{ll}S_{1} G & S_{1} G_{d}\end{array}\right]$ was applied to select 4 temperatures to control the distillation column. The temperatures selected are on stages $9,16,24$, and 33 . 


\subsubsection{Step 2: perfect disturbance rejection}

For the distillation column example with (from step 1)

$$
\left[\begin{array}{l}
c_{1} \\
c_{2}
\end{array}\right]=H\left[\begin{array}{c}
T_{9} \\
T_{16} \\
T_{24} \\
T_{33}
\end{array}\right]
$$

zero disturbance loss is obtained with

$$
H=\tilde{G}_{1} \tilde{G}^{\prime-1}=\left[\begin{array}{cccc}
-0.7068 & 0.0444 & 0.0911 & -0.0663 \\
0.0442 & -0.0376 & -0.0766 & 0.6903
\end{array}\right]
$$

As the $H$-matrix is not unique, we have normalized it so that its 2-norm is equal to 1 , i.e., $\|H\|_{2}=1$.

The maximum loss, which in this case is caused by measurement error only, is equal to 0.822 (calculated by exact local method). Interestingly, this combination gives a maximum loss larger than the obtained by the best two single temperatures (see Table 1). This happens because the measurement selection in Step 1 in non-optimal.

\subsubsection{Step 2: exact local method}

Eq. (26), which also accounts for implementation error, was used to obtain the optimal combination of the same four temperatures, as in Eq. (27). The resulting $H$-matrix is

$$
H=\left[\begin{array}{cccc}
0.4751 & 0.3980 & -0.2433 & -0.0474 \\
-0.0770 & 0.1070 & -0.2456 & -0.6905
\end{array}\right]
$$

which gives a maximum loss of 0.582 . In this case, the loss due to disturbance is 0.370 (since we do not have perfect disturbance rejection) and due to implementation error is 0.516. Again, the maximum loss is larger than the best combination of two temperatures (0.530).

\subsection{Exact local method with four measurements}

Thus, the two-step procedure fails to give a good result in this case, so we here use the exact local method to find the truly optimal combination. The best set of 4 temperatures $(10,11,31$, and 32) were found evaluating the exact local method for all possible combinations $\left(\frac{41 \times 40 \times 39 \times 38}{4 \times 3 \times 2}=101270\right.$ combinations), but Kariwala ${ }^{6}$ derived a branch and bound algorithm that could alternatively have been used to find the best solution in a more efficient way. 
It is interesting to note that it is optimal to select neighboring temperatures close to the location of the optimal with only two measurements $\left(T_{12}-T_{30}\right)$. Apparently, this is to reduce the effect of measurement error. The resulting optimal combination using Eq. (26) is

$$
H=\left[\begin{array}{cccc}
0.4087 & 0.4962 & 0.1880 & 0.1349 \\
-0.2269 & -0.2980 & -0.4831 & -0.3994
\end{array}\right]
$$

The estimated maximum loss in this case is 0.440 (0.302 due to disturbances and 0.407 due to implementation error), which is now smaller than the best combination of two temperatures (0.530).

Note that $H$ is not unique, and by choosing $D=\left[\begin{array}{cc}1 & 0.389 \\ -0.555 & -1\end{array}\right]$, we find that another optimal combination matrix is

$$
H=\left[\begin{array}{cccc}
0.320 & 0.380 & 0 & -0.020 \\
0 & 0.023 & 0.379 & 0.325
\end{array}\right]
$$

and we see that the optimal combination is approximately to control a combination of $T_{10}$ and $T_{11}$ and a combination of $T_{31}$ and $T_{32}$.

\subsection{Combining all measurements}

A two-step procedure is not needed if we combine all 41 temperatures. In this case, the matrix $H$ can be calculated using the analytical solution for the exact local method, Eq. (26), resulting in a maximum loss of 0.226 (0.067 due to disturbance and 0.216 to implementation error), which is the lowest possible.

The results for all the cases studied are summarized in Table 2.

Table 2: Composition deviation $\Delta X$ for various configurations: summary.

\begin{tabular}{l|ccc}
\hline & \multicolumn{3}{|c}{ Loss } \\
\cline { 2 - 4 } Controlled variables $c_{1}-c_{2}$ & Disturbance & Meas. Noise & Total \\
\hline$T_{12}-T_{30}$ & 0.376 & 0.513 & 0.530 \\
$H\left[T_{9}, T_{16}, T_{24}, T_{33}\right]((28)$ from Eq. 25$)$ & 0 & 0.822 & 0.822 \\
$H\left[T_{9}, T_{16}, T_{24}, T_{33}\right]((29)$ from Eq. 26$)$ & 0.370 & 0.516 & 0.582 \\
$H\left[T_{10}, T_{11}, T_{31}, T_{32}\right]((30)$ from Eq. 26$)$ & 0.302 & 0.407 & 0.440 \\
$H[$ All temperatures] (Eq. 26) & 0.067 & 0.216 & 0.226 \\
\hline
\end{tabular}

\section{Conclusions}

In this paper, we evaluated a systematic way of selecting secondary controlled variables using the maximum scaled gain rule. 
The maximum gain rule involving $S_{1} G J_{u u}^{-1 / 2}$, see Eq. (6), is preferred if we can obtain easily the Hessian $J_{u u}$. The results are very close to the optimum and do not require the evaluation of all possible candidates. So, for large systems, the maximum gain rule is a preferred choice.

The simplified maximum gain rule involving $S_{1} G$, see Eq. (13), is the easiest to apply because it does not require an evaluation of the Hessian $J_{u u}$. This rule is usually good but unfortunately it can give wrong result for ill-conditioned systems, like some distillation columns (see Table 1). This problem could be avoided by choosing a different set of base variables $u$ such that $J_{u u}$ is close to an unitary matrix.

The output scaling $\left(S_{1}\right)$ is an important factor, especially when we have different kinds of candidate controlled variables like temperatures and flows.

The selection of combinations of measurements to minimize the effect of disturbances and implementation error was also considered. A two-step approach may be used, where in the first step the measurements are selected and, in the second, the combination of the selected measurements is obtained. The results show that this methodology, although it is not exact, is able to obtain a control structure with a small effect of implementation error (step 1) and disturbances (step 2) in the primary variables.

\section{References}

(1) Yi, C.K.; Luyben, W. L. Evaluation of plant-wide control structures by steady-state disturbance sensitivity analysis. Ind. Eng. Chem. Res. 1995, 34(7), 2393

(2) Mellefont, D.J.; Sargent, R. W. H. Selection of measurements for optimal feedback control. Ind. Eng. Chem. Proc. Des. Dev. 1978, 17(4), 549 .

(3) Moore, C. F. Selection of controlled and manipulated variables. Practical Distillation Control, W.L. Luyben (ed.): Van Nostrand Reinhold, 1992.

(4) Chmielewski, D. J.; Palmer, T.; Manousiouthakis, V. On the theory of optimal sensor placement. AIChE J. 2002, 48(5), 1001.

(5) Bian, S.; Henson, M. A. Measurement selection for on-line estimation of nonlinear wave models for high purity distillation columns. Chem. Eng. Sci. 2006, 61, 3210. 
(6) Kariwala, V. Optimal measurement combination for local selfoptimizing control. Ind. Eng. Chem. Res. 2007, 46(11), 3629.

(7) Skogestad, S. Plantwide control: the search for the self-optimizing control structure. J. Proc. Cont. 2000, 10(5), 487.

(8) Morari, M.; Arkun, Y.; Stephanopoulos, G. Studies in the synthesis of control structures for chemical processes. part i. formulation of the problem. process decomposition and the classification of the control task. analysis of the optimizing control structures. AIChE J. 1980, 26(2), 220.

(9) Findeisen, W.; Bailey, F. N.; Brdys, M.; Malinowski, K.; Tatjewski, P.; Wozniak, A. Dynamics and Control of Continuous Distillation Units: John Wiley and Sons, New York, 1980.

(10) Halvorsen, I.J.; Skogestad, S.; Morud, J.; Alstad, V. Optimal selection of controlled variables. Ind. Eng. Chem. Res. 2003, 42(14), 3273.

(11) Tolliver, T. L.; McCune, L. C. Finding the optimum temperature control trays for distillation-columns. Instrum. Tech. 1980, $27(9), 75$.

(12) Hori, E.S.; Skogestad, S.; Alstad, V. Perfect steady-state indirect control. Ind. Eng. Chem. Res. 2005, 44(4), 863.

(13) Alstad, V.; Skogestad, S. Null space method for selecting optimal measurement combinations as controlled variables. Ind. Eng. Chem. Res. 2007, 46(3), 846 .

(14) Alstad, V.; Skogestad, S.; Hori, E. S. Extended nullspace method for selecting measurement combinations as controlled variables for nearoptimal steady-state operation. Submitted to J. Proc. Cont. 2007

(15) Cao, Y.; Rossiter, D.; Owens, D. H. Globally optimal control structure selection using branch and bound method. Preprints of DYCOPS'5 1998: Corfu, Greece.

(16) Skogestad, S.; Postlethwaite, I. Multivariable feedback control - analysis and design - 2nd Edition. John Wiley and Sons, London, UK, 2005.

(17) Hori, E.S.; Skogestad, S. Selection of control structure and temperature location for two-product distillation columns. Chem. Eng. Res. Des. 2007, 85(A3), 293. 
(18) Shinskey, F.G. Distillation control 2nd Edition. McGraw-Hill: New York, 1984.

(19) Skogestad, S. Dynamics and control of distillation columns: a tutorial introduction. Chem. Eng. Res. Des. 1997, 75(A6), 539.

(20) Hori, E.S.; Skogestad, S.; Al Arfaj, M. A. distillation columns. Distillation and Absorption 2006: London, UK.

(21) Luyben, W. L. Effect of feed composition on the selection of control structures for high-purity binary distillation. Ind. Eng. Chem. Res. 2005, 44(20), 7800 .

(22) Yu, C. C.; Luyben, W.L. Use of multiple temperatures for the control of multicomponent distillation columns. Ind. Eng. Chem. Proc. Des. Dev. 1984, 23(3), 590.

(23) Chew, M.; Jones, W. E.; Wilson, J. A. Distillation column control using the whole temperature profile. Distillation and Absorption 2006: London, UK. 


\section{List of figure captions}

- Figure 1: Block diagram of indirect control with selection of measurements.

- Figure 2: Distillation column.

- Figure 3: Optimal variations and total span (output scaling $S_{1}$ ).

- Figure 4: Comparison of composition deviation estimates $\left(S_{1} G J_{u u}^{-1 / 2}\right.$, $S_{1} G$, and exact local method). 